\title{
BMJ Open Efficacy and safety of xuezhikang once per day versus two times per day in patients with mild to moderate hypercholesterolaemia (APEX study): a protocol for a multicentre, prospective randomised controlled, open-label, non- inferiority study
}

\author{
Zexuan Wu, ${ }^{1,2}$ Dexi Wu, ${ }^{1,2}$ Jingzhou Jiang, ${ }^{1,2}$ Ailan Chen, ${ }^{3}$ Dong-Dan Zheng, \\ Jianhao Li, ${ }^{5}$ Yugang Dong, ${ }^{1,2}$ Yili Chen (D) ${ }^{1,2}$
}

To cite: Wu Z, Wu D, Jiang J, et al. Efficacy and safety of xuezhikang once per day versus two times per day in patients with mild to moderate hypercholesterolaemia (APEX study): a protocol for a multicentre, prospective randomised controlled, open-label, non-inferiority study. BMJ Open 2020;10:e034585. doi:10.1136/ bmjopen-2019-034585

- Prepublication history and additional material for this paper are available online. To view these files, please visit the journal online (http://dx.doi. org/10.1136/bmjopen-2019034585).

ZW and DW contributed equally.

Received 26 September 2019 Revised 21 February 2020 Accepted 09 April 2020

Check for updates

(C) Author(s) (or their employer(s)) 2020. Re-use permitted under CC BY-NC. No commercial re-use. See rights and permissions. Published by BMJ.

For numbered affiliations see end of article.

Correspondence to

Dr Yili Chen;

yilichen2018@126.com

\section{ABSTRACT}

Introduction Reduction in low-density lipoprotein cholesterol (LDL-C) improves clinical outcomes in patients with coronary artery disease. However, rates of lipid-lowering medication adherence are far from ideal. Reducing dosage frequency from multiple dosing to once-daily dosing may improve patients' medication adherence. Xuezhikang (XZK), an extract of Chinese red yeast rice, contains a family of naturally occurring statins and is traditionally prescribed as $600 \mathrm{mg}$ two times per day. A comParative Efficacy study of XZK (APEX study) is designed to test the hypothesis that XZK prescribed $1200 \mathrm{mg}$ once per day (OD group) is non-inferior to $600 \mathrm{mg}$ two times per day (TD group) in patients with hypercholesterolaemia.

Methods and analysis The APEX study is a multicentre, prospective randomised controlled, open-label, noninferiority study. We plan to recruit 316 patients aged $\geq 18$ years with a diagnosis of mild to moderate hypercholesterolaemia for primary prevention. Patients will be randomised (1:1) to OD group and TD group. The OD group take XZK $1200 \mathrm{mg}$ once per day after dinner while TD group take a traditional dose of $600 \mathrm{mg}$, two times per day after meals. Participants will have an 8week medication period and be followed up at weeks 0 , 4 and 8 . The primary end point is the mean percentage change from baseline to week 8 in serum LDL-C.

Secondary end points are safety and lipid-lowering effect on other lipoproteins and compliance. Data analyses will be on the intention-to-treat principle using non-inferiority analysis.

Ethics and dissemination The research had been approved by the Clinical Research and Laboratory Animal Ethics Committee of the First Affiliated Hospital, Sun Yatsen University ((2017)286). The results will be reported through peer-reviewed journals, seminars and conference presentations.

Trial registration number ChiCTR-IIR-17013660.
Strengths and limitations of this study

- One of the first, well-designed randomised controlled trial studying non-inferiority of xuezhikang (XZK), a red yeast rice, prescribed $1200 \mathrm{mg}$ once per day versus $600 \mathrm{mg}$ two times per day in patients with mild to moderate hypercholesterolaemia.

- May improve patients' adherence to lipid-lowering medication.

- A multicentre study.

- Non-blinded.

- The lipid-lowering efficacy of every single ingredient within XZK can hardly be evaluated in our study.

\section{INTRODUCTION}

Cardiovascular diseases (CVDs) have become the leading cause of death. About 17.9 million people die each year from CVDs, estimating $31 \%$ of all deaths worldwide. ${ }^{1}$ Hyperlipidaemia, primarily hypercholesterolaemia, is one of the most important risk factors. Reduction in low-density lipoprotein cholesterol (LDL-C) greatly improves clinical outcomes in patients with CVDs. ${ }^{2}$ Reduction in LDL-C reduces the rate ratio for CVDs similarly in primary and secondary prevention populations. ${ }^{34}$ Despite the benefits of statin, patients discontinued statin due to various reasons including side effects (mainly liver dysfunction, myalgia and myopathies).$^{5}$ One recent study in the UK showed that the adherence of patients with CVDs receiving statin is only $68 \%-72 \%$. About $43 \%$ of patients discontinued their medication 1 year after initiation. ${ }^{6}$ Poor statin adherence was associated with increased risk of CVDs and mortality. ${ }^{7}$ 
Thus improving adherence to lipid-lowering medication is critical to achieving optimal clinical outcomes.

Over the past few years, a growing attention has been devoted to the management of dyslipidaemia by use of dietary supplements. Xuezhikang (XZK) is an extract of Chinese red yeast rice (RYR, in general Monascus purpureus) used in China for over 2000 years. It contains a family of naturally occurring statins, one of which is lovastatin (monacolin $\mathrm{K}$ ), in addition to unsaturated fatty acids, ergosterol, amino acids, flavonoids, alkaloid, trace element and other substances. ${ }^{89}$ The effects of XZK on the cholesterol concentration might be caused by the additive and/or synergistic effects of lovastatin (monacolin K) with other monacolins and substances in XZK. ${ }^{10}$ Although $1200 \mathrm{mg}$ of XZK contains only $10 \mathrm{mg}$ of lovastatin, daily treatment with XZK 1200 or $2400 \mathrm{mg}$ for 12 weeks significantly reduced LDL-C $(\sim 27 \%)$, non-highdensity lipoprotein cholesterol (non-HDL-C, $\sim 24 \%$ ) and other atherogenic lipids and lipoproteins, and was safe and well tolerated in both American and Chinese patients with moderately elevated LDL-C. ${ }^{11}$ Due to the short halflife period of only $2.15 \pm 0.16$ hours, XZK was prescribed as $600 \mathrm{mg}$ two times per day. ${ }^{10}$ However, previous metaanalyses suggested that reducing dosage frequency from multiple dosing to once-daily dosing improved adherence to therapies among patients with acute and chronic diseases. $^{12}$ Considering despite even shorter half-life period of $1.22 \pm 0.04$ hours, lovastatin was prescribed only once daily after dinner, ${ }^{10}$ we infer that XZK can be also taken once per day after dinner and is non-inferior to the typically two times per day regimen in lipid-lowering efficacy. However, no related clinical trials have been conducted.

Therefore, A comParative Efficacy study of XZK (APEX study) is designed as a non-inferiority study to assess the efficacy and safety of XZK $1200 \mathrm{mg}$ once per day versus $600 \mathrm{mg}$ two times per day in lipid reduction in patients with hypercholesterolaemia.

\section{METHODS AND ANALYSIS}

\section{Study design}

The APEX study is an ongoing, multicentre, prospective, open-label, non-inferiority, randomised controlled trial (RCT) designed to assess the efficacy and safety of XZK $1200 \mathrm{mg}$ once per day compared with $600 \mathrm{mg}$ two times per day in the patients with mild to moderate hypercholesterolaemia for primary prevention.

\section{Study hypothesis}

The administration of XZK $1200 \mathrm{mg}$ once per day is non-inferior to $600 \mathrm{mg}$ two times per day in terms of efficacy and safety in patients with mild to moderate hypercholesterolaemia.

\section{Study population, eligibility criteria and recruitment}

Patients aged $\geq 18$ years with mild to moderate hypercholesterolaemia in primary prevention of arteriosclerotic CVD

\section{Box 1 Eligibility criteria}

Inclusion criteria

- Age $\geq 18$ years.

- LDL-C level $\geq 3.0 \mathrm{mmol} / \mathrm{L}$ (116 mg/dL), but $<4.9 \mathrm{mmol} / \mathrm{L}$ (190 mg/ $\mathrm{dL}$ ) and TG level $<4.52 \mathrm{mmol} / \mathrm{L}(400 \mathrm{mg} / \mathrm{dL})$.

- Body mass index $\geq 18.5$ but $<30.0 \mathrm{~kg} / \mathrm{m}^{2}$.

- Female patients must be at least 12 months postmenopause, or sterilised at least 3 months before the study begins, or have a negative pregnancy test at screening and agree to use reliable contraceptive methods during the study period until 1 month after the study ends.

- Willing to follow a TLC diet.

- Willing to provide written informed consent.

Exclusion criteria

- History of ASCVD including previous ACS (MI or unstable angina), stable angina, coronary revascularisation ( $\mathrm{PCl}, \mathrm{CABG}$ and other arterial revascularisation procedures), stroke, TIA or peripheral arterial disease.

- Uncontrolled hypertension (systolic blood pressure $\geq 180 \mathrm{~mm} \mathrm{Hg}$ and/ or diastolic blood pressure $\geq 110 \mathrm{~mm} \mathrm{Hg}$ ) at screening.

- Abnormal liver function test at baseline (ALT or AST >1.5×ULN).

- Elevated CK at baseline (CK > ULN).

- Known renal dysfunction or elevated serum creatinine levels beyond the range of ULN at baseline.

- DM patients except those young patients (T1DM $<35$ years; T2DM $<50$ years) with DM duration $<10$ years and without other risk factors.

- Other endocrine diseases that might influence the levels of lipid and lipoprotein such as hypothyroidism.

- Allergy to red yeast rice.

- Participation in other clinical studies within 4 weeks before the first visit.

- Pregnant or lactating women.

- Other situations that may prevent the participant from participating in the study (eg, active psychosis).

Values measured with 1 month before obtaining informed consent can be used for assessing eligibility.

ACS, acute coronary syndrome; ALT, alanine aminotransferase; ASCVD, atherosclerotic cardiovascular disease; AST, aspartate aminotransferase; CABG coronary artery bypass grafting; $\mathrm{CK}$, creatine kinase; DM, diabetes mellitus; LDL-C, low-density lipoprotein cholesterol; MI, myocardial infarction; PCI, percutaneous coronary intervention; TG, triglyceride; TIA, transient ischaemia attack; TLC, therapeutic lifestyle changes; T1DM, type 1 diabetes mellitus; T2DM, type 2 diabetes mellitus; ULN, upper limit of normal.

(ASCVD) are eligible for participation. The LDL-C level is defined $\geq 3.0 \mathrm{mmol} / \mathrm{L}(116 \mathrm{mg} / \mathrm{dL})$ but $<4.9 \mathrm{mmol} / \mathrm{L}$ $(190 \mathrm{mg} / \mathrm{dL})$ and triglyceride $(\mathrm{TG})<4.52 \mathrm{mmol} / \mathrm{L}(400$ $\mathrm{mg} / \mathrm{dL}$ ). ${ }^{4}$ The body mass index (BMI) is $\geq 18.5 \mathrm{~kg} / \mathrm{m}^{2}$ but $<30.0 \mathrm{~kg} / \mathrm{m}^{2}{ }^{13}$ Patients must be willing to follow a therapeutic lifestyle changes (TLC) diet through the study period. ${ }^{14}$ The exclusion criteria are detailed in box 1 . Patients with a history of ASCVD including previous acute coronary syndrome (ACS), stable angina, coronary revascularisation, stroke, transient ischaemia attack (TIA) or peripheral arterial disease are excluded. Patients with a total cardiovascular risk of high to very high risk who require lower LDL-C goal were also excluded, including those with uncontrolled hypertension (systolic blood 

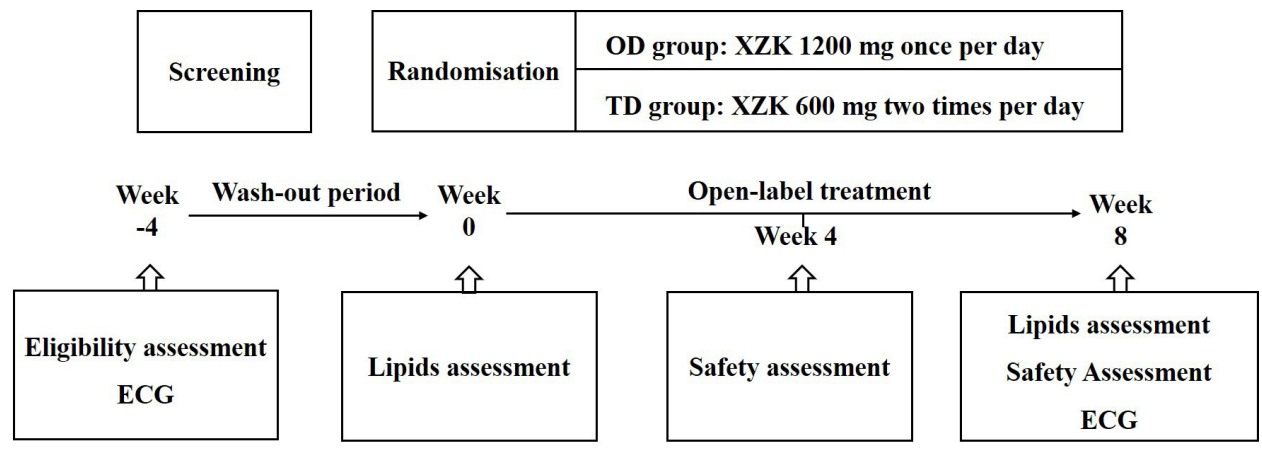

Figure 1 Study design. OD, once per day; TD, two times per day; XZK, xuezhikang.

pressure $\geq 180 \mathrm{~mm} \mathrm{Hg}$ and/or diastolic blood pressure $\geq 110 \mathrm{~mm} \mathrm{Hg}$ ) at screening or all diabetes mellitus (DM) patients except those young patients (type 1 DM (T1DM) $<35$ years old; type 2 DM (T2DM) $<50$ years old) with DM duration $<10$ years and without other risk factors. ${ }^{4}$ Patients with liver dysfunction, renal dysfunction as well as elevated creatine kinase (CK) were also excluded. Study candidates will be assessed for eligibility within 4 weeks prior to enrolment (figure 1).

The study is now conducted in eight medical centres in Guangdong province in China. Patients will be recruited by local physicians using posters and advertisement in the hospital and nearby communities. Posters and advertisement contain brief introductions about the study and the contact information of the investigators. The study will be detailed explained to the potential participants and informed written consent will be obtained prior to enrolment.

\section{Randomisation, allocation and blinding}

Random sequence is computer generated by an independent statistician and stratified by centre. A permuted block randomisation with a block size of either 4 or 6 was used to minimise the chance that investigators could guess the treatment assignment. The randomisation numbers are kept in opaque sealed envelopes. Enrolment and randomisation will be handled at the sites. Patients and investigators will be informed about randomisation result prior to initiation of treatment. Participants fulfilling the eligibility criteria will be randomly allocated in a 1:1 ratio to either once per day (OD) group or two times per day (TD) group.

Because the study aims to compare the efficacy between different frequency of administration, we adopted an open-label design. Nonetheless, laboratory technicians performing blood/urine tests will be blinded to treatment allocation, thus reducing observer bias to the greatest extent.

\section{Intervention}

The OD group is administered with XZK (produced by Beijing Beida Weixin Biotechnology Co, China) at a dose of $1200 \mathrm{mg}$ (four capsules, $300 \mathrm{mg}$ per capsule) once per day orally after dinner while the TD group taking a traditional dose of $600 \mathrm{mg}$ (two capsules) two times per day orally after meals. All the patients will be treated for 8 weeks. No treatment with other antihyperlipidaemic agents was allowed during the whole study period. However, other kinds of medications were taken as needed.

\section{Outcomes}

\section{Primary outcome}

The primary outcome is the mean percentage change in serum LDL-C from baseline to week 8 in the intention-totreat (ITT) population. ${ }^{15}$

\section{Secondary outcomes}

The secondary outcomes include the mean percentage change from baseline to week 8 in other lipoproteins including total cholesterol (TC), TG, LDL-C, HDL-C, non-HDL-C, apolipoprotein E (Apo E), apolipoprotein B (Apo B), apolipoprotein A-I (Apo A-I), lipoprotein (a) $(\operatorname{Lp}(\mathrm{a}))$ and the ratio of Apo A-I to Apo B. The proportion of patients achieving target LDL-C level of 3.0 $\mathrm{mmol} / \mathrm{L}(116 \mathrm{mg} / \mathrm{dL}), 2.6 \mathrm{mmol} / \mathrm{L}(100 \mathrm{mg} / \mathrm{dL})$ and $1.8 \mathrm{mmol} / \mathrm{L}(70 \mathrm{mg} / \mathrm{dL})$ at the end of week 8 will also be evaluated, respectively. Patients compliance will also be calculated as another secondary outcome.

Safety will be assessed based on adverse events reported throughout the study period, vital signs, physical examination, 12-lead ECG, clinical laboratory tests and the use of rescue medication (details listed in box 2).

\section{Study outlines}

Screening visit (week -4)

Schedule of enrolment and assessments are listed in table 1 . Subjects will be evaluated at the screening visit 4 weeks prior to enrolment through a complete medical history, vital signs, physical examination, 12-lead ECG, eligibility laboratory tests and a review of concomitant medications. Eligibility laboratory variables include urine pregnancy testing for women with childbearing potential, lipid profiles, fasting blood glucose, aspartate aminotransferase (AST), alanine aminotransferase (ALT), CK and creatinine. Variables measured with 4 weeks before obtaining informed consent can be used for eligibility assessment. TLC diet guidance will be given throughout the study period. 


\section{Box 2 Efficacy and safety endpoints}

Primary endpoint

- The mean percentage change in serum LDL-C from baseline to week 8 in ITT population.

\section{Secondary endpoint}

- The mean percentage change from baseline to week 8 in ITT population.

TC.

TG.

HDL-C.

Non-HDL-C.

Apo E.

Apo A-I.

Apo B.

Lp(a).

Ratio of Apo A-I to Apo B.

- Proportion of patients reaching LDL-C target of $<3.0 \mathrm{mmol} / \mathrm{L}$ (116 $\mathrm{mg} / \mathrm{dL}$ ) at week 8.

- Proportion of patients reaching LDL-C target of $<2.6 \mathrm{mmol} / \mathrm{L}$ (100 $\mathrm{mg} / \mathrm{dL}$ ) at week 8.

- Proportion of patients reaching LDL-C target of $<1.8 \mathrm{mmol} / \mathrm{L}$ (70 $\mathrm{mg} / \mathrm{dL}$ ) at week 8 .

- Patients' compliance.

Safety endpoints

- Treatment-emergent adverse event.

- Adverse event of special interest including liver dysfunction, statinassociated muscle symptom, myalgia, myopathies, rhabdomyolysis, renal dysfunction and newly diagnosed DM.

- Vital signs.

- Physical examination.

- ECG.

- Clinal laboratory tests.

Apo, apolipoprotein; DM, diabetes mellitus; HDL-C, high-density lipoprotein cholesterol; ITT, the intention-to-treat; LDL-C, low-density lipoprotein cholesterol; Lp(a), lipoprotein(a); TC, total cholesterols; TG, triglyceride.

\section{Wash-out period}

Patients who are taking lipid-lowering drugs at screening will be asked to start a 4-week wash-out period, in which they will discontinue the lipid-lowering drugs and follow the guideline for TLC diet and lifestyle control. The serum lipid profiles will be re-evaluated for eligibility before randomisation.

\section{Baseline visit (week 0)}

Baseline lipid assessment will be conducted at baseline visit. Participants without wash-out can use the lipid levels assessed at screening as baseline data. Participants who meet the eligibility criteria will be randomly assigned (1:1) to receive XZK $1200 \mathrm{mg}$ once per day or $600 \mathrm{mg}$ two times per day. Patients will be informed of the intention to monitor pill-taking compliance.

Follow-up visit (week 4)

Patients will be followed up at week 4 to assess safety and tolerability. Vital signs, physical examination, blood analyses and the use of rescue medication will be recorded. Blood analyses for safety include ALT, AST, CK, creatinine
Table 1 Schedule of assessments

\begin{tabular}{lllll} 
Visit number & V1 & V2 & V3 & V4 \\
Week & -4 & 0 & 4 & 8 \\
Day & $-28 \pm 2$ & 0 & $28 \pm 2$ & $56 \pm 2$ \\
$\begin{array}{l}\text { Informed consent } \\
\begin{array}{l}\text { Inclusion/exclusion } \\
\text { criteria }\end{array}\end{array}$ & $\times$ & $\times$ & & \\
\hline
\end{tabular}

$\begin{array}{lllll}\text { Medical history } & \times & \times & & \\ \begin{array}{l}\text { Concomitant } \\ \text { medication }\end{array} & \times & \times & \times & \times\end{array}$

$\begin{array}{lllll}\text { Vital signs } & \times & \times & \times & \times \\ \text { Physical examination } & \times & \times & & \times \\ \text { 12-lead ECG } & \times & & & \times\end{array}$

Laboratory tests for $x$

eligibility*

\begin{tabular}{|c|c|c|c|c|}
\hline TLC diet guidance & $x$ & $x$ & $x$ & $x$ \\
\hline Lipids assessment & & $\times \neq$ & & $x$ \\
\hline
\end{tabular}

for efficacy

\begin{tabular}{|c|c|c|c|c|}
\hline $\begin{array}{l}\text { Laboratory tests for } \\
\text { safety§ }\end{array}$ & & & $x$ & $x$ \\
\hline $\begin{array}{l}\text { Dispense study } \\
\text { medication/collect } \\
\text { empty study }\end{array}$ & & $x$ & $x$ & \\
\hline $\begin{array}{l}\text { Compliance } \\
\text { evaluation }\end{array}$ & $x$ & $x$ & $x$ & $x$ \\
\hline Adverse events & $\times$ & $x$ & $x$ & $x$ \\
\hline
\end{tabular}

Values measured with 4 weeks before obtaining informed consent can be used for assessing eligibility.

*Laboratory tests for eligibility include serum lipids, fasting blood glucose, alanine aminotransferase (ALT), aspartate aminotransferase (AST), creatine kinase (CK), creatinine and urine pregnancy testing for women with childbearing potential. TTLC: therapeutic lifestyle changes.

$\ddagger$ Patients who do not need wash-out can use the lipids results at screening as baseline levels and do not need to reassess lipids level at randomisation.

§Laboratory tests for safety include ALT, AST, CK, creatinine and fasting glucose.

and fasting glucose. Any adverse events during the intervening period since screening/baseline visit will be recorded. Study drug dispensed at screening/baseline visit will be collected for compliance calculation.

\section{Final evaluation (week 8)}

Final evaluations will be conducted 8 weeks following the baseline assessment. Vital signs, physical examination, 12-lead ECG, blood analyses and adverse events will be assessed. Blood analyses include ALT, AST, CK, creatinine and fasting glucose for safety assessments and a fasting lipid profile for efficacy assessment.

\section{Sample collection and laboratory measurements}

Blood sample collection is scheduled at the day of screening, randomisation and then during two follow-up visits, after 4 and 8 weeks. Patients are advised to avoid 
excessive physical effort within 48 hours preceding the blood collection and be fasting at least 12 hours prior to the test. Blood samples are collected into evacuated tubes by using aseptic precautions and clot for $30 \mathrm{~min}$ in a vertical position at room temperature. Serum is then separated by centrifugation for $10 \mathrm{~min}$ at $3000 \times \mathrm{g}$ at room temperature. Following the centrifugation, routine laboratory measurements are performed including fasting blood glucose, lipid profile (TC, TG, LDL-C, HDL-C, Apo E, Apo A-I, Apo B, Lp(a)), AST, ALT, CK and creatinine using commercial kits. TC and TG are evaluated by the enzymatic method and glycerol-3-phosphate oxidase (GPO) and peroxidase (POD) method ${ }^{16}$ separately. LDL-C and HDL-C are measured by the direct method. Non-HDL-C is calculated as TC minus HDL-C. Apo E, Apo A-I, Apo B and Lp(a) are determined by turbidimetric inhibition immunoassay. All measurements are performed on the Beckman Coulter AU5800 chemistry analyser. Urine samples will also be collected at screening for urine pregnancy test for women with childbearing potential.

Laboratory measurements are performed at the Department of Laboratory Medicine of each site, holding national and international procedures for external quality control.

\section{Compliance evaluation}

Compliance will be measured by pill taking (total doses taken divided by total prescribed doses). In assessing compliance, the denominator (total prescribed doses) will be calculated over the actual duration the subject was in the study. Subjects will be informed of the intention to monitor pill-taking compliance in the informed consent form.

\section{Adverse events and safety}

Adverse events will be assessed at each study visit. Given the nature of the intervention, liver dysfunction, statinassociated muscle symptom (SAMS), myalgia, myopathies, rhabdomyolysis, renal dysfunction as well as newly diagnosed DM are adverse events of special interest. The diagnosis and management of the statin associated adverse events will follow the scientific statement from the American Heart Association. ${ }^{17}$ To be noted, for patients with muscle complaints, drucebo effect should also be considered. ${ }^{18}$ Therefore, SAMS Clinical Index (SAMS-CI) score will be used to evaluate the likelihood that the patients' muscle symptoms are truly statin-related. ${ }^{19}$ For those with low SAMS-CI score, other causes affecting musculoskeletal system, history of medication-related side effects, depression and anxiety will further be evaluated before discontinuation of XZK.

All adverse events will be recorded in the case report forms from the point of informed consent. All serious adverse events will be assessed by the chief investigators in terms of seriousness and causality and reported to the ethics committee in accordance with the International Council for Harmonisation of Technical Requirements for Registration of Pharmaceuticals for Human Use Good Clinical Practice (GCP) guidelines. ${ }^{20}$

\section{Data management and monitoring}

Data collection will be performed by trained local research staff at each of the trial visits in the form of case report forms. This will then be entered into the APEX trial database, which is housed in clinical research unit in Department of Cardiology in the First Affiliated Hospital, Sun Yat-sen University and supervised by the trial data manager. Data from the APEX study will be transferred securely to the Research Data Deposit at http://www. researchdata.org.cn/, where it can be accessed and traced securely via a specialised administration panel by members of the research team using an encrypted password.

The APEX study will undergo monitoring for regulatory compliance in accordance with the GCP guidance via the trial steering committee, which independently monitors progress and conduct of the trial and will also provide advice on the continuation, termination or amendments to the trial protocol. The study is sponsored by Beijing Beida Weixin Biotechnology Co and will be subjected to regular monitoring visits and audits.

\section{Study status}

Patient recruitment was started in November 2018 according to the protocol of version 2.0, 07 July 2018. The original inclusive LDL-C range was 130-220 mg/dL and BMI between 18 and $36 \mathrm{~kg} / \mathrm{m}^{2}$. Patients with recent CVD as well as newly and uncontrolled DM were excluded. Up to February 2020, 10 patients were enrolled and 1 patient (No. 08009) withdrew her informed consent due to diarrhoea symptom (online supplementary file 1). However, considering the LDL-C reduction intensity of about $27 \%$ in $\mathrm{XZK}^{12}$ and a stricter LDL-C goal for high to very high cardiovascular (CV) risk patients according to the 2019 European Society of Cardiology and European Atherosclerosis Society guidelines, ${ }^{4}$ we revised the inclusion criteria and excluded the patients with high to very high CV risk (protocol version 3.0, 07 February 2020). All the 10 patients will be included in the safety analysis. One patient with a history of coronary heart disease and DM (No. 08001), and another patient with a high baseline LDL-C level of $203 \mathrm{mg} / \mathrm{dL}$ (No. 08004) will be eliminated in the final efficacy analysis. The baseline LDL-C range of the other eight patients were all within the range of $116-190 \mathrm{mg} / \mathrm{dL}$. And the BMI range of the 10 patients were all within the range of 18.5-30 $\mathrm{kg} / \mathrm{m}^{2}$. In addition, since our study is a non-inferiority of different dosing on the efficacy of treatment, compliance is our critical focus and will be evaluated in every patient throughout the study. However, in our previous protocol (version 2.0,07 July 2018), we did not include it as one of the secondary endpoints. In our revised protocol (version 3.0, 07 February 2020), we added as one of the secondary endpoints. We had obtained the ethics approval for the revisions. The revisions add no harm to the patients and 
will not influence the statistical power of the study. The study is expected to conclude in December 2020.

\section{Patient and public involvement}

Patients were not involved in the design or in the recruitment to and conduct of the study, nor in the assessment of burden of the intervention. Results will be disseminated to participants on request.

\section{STATISTICAL CONSIDERATION}

\section{Sample size assumptions}

Sample size calculation is based on the primary outcome, namely change in LDL-C from baseline to follow-up at 8 weeks. According to previous studies, the mean percentage change of LDL-C level is $27.7 \% \pm 12.2 \%$ after 8 weeks of treatment with XZK $600 \mathrm{mg}$ two times per day and $25.1 \%$ after 8 weeks of XZK $1200 \mathrm{mg}$ once per day treatment. ${ }^{1521}$ We therefore assumed the reduction of LDL-C as $27 \%$ and $25 \%$ in the two groups and both SD as $12 \%$. A non-inferiority threshold of $6 \%$ is considered according to previous lipid studies. ${ }^{22}$ A sample size of 142 per group will yield $80 \%$ power to obtain a significant difference between the two groups, using a two-sample t-test at a one-sided 0.025 level of significance. To account for an approximately $10 \%$ dropout rate, a total of 316 subjects (158 subjects for each group) will be enrolled.

\section{Statistical analysis}

The primary analysis will evaluate the ITT population (all randomised subjects). All efficacy and demographic analyses will be performed on the ITT and per-protocol population (all randomised subjects completing the 8 weeks treatment). Safety analyses will be performed on all randomised subjects receiving $\geq 1$ dose of study medication. Missing data will be handled according to the complete-case analysis principle, based on the availability of the data needed to evaluate the primary outcome.

The primary analysis is the statistical comparison of mean percentage changes of LDL-C between the OD group and the TD group.

The analysis will be repeated on secondary efficacy parameters including: mean percentage changes from baseline to week 8 in TC, TG, HDL-C, non-HDL-C, Apo E, Apo B, Apo A-I, Lp(a) as well as the ratio of Apo-B to Apo A-I between the two groups. The proportion of patients reaching the target of $3.0 \mathrm{mmol} / \mathrm{L}(116 \mathrm{mg} / \mathrm{L})$, $2.6 \mathrm{mmol} / \mathrm{L}(100 \mathrm{mg} / \mathrm{dL})$ and $1.8 \mathrm{mmol} / \mathrm{L}(70 \mathrm{mg} / \mathrm{dL})$ as well as participant compliance will also be compared at the end of week 8.

Continuous variables will be summarised by median and IQR or mean \pm SD. Categorical variables will be expressed as frequencies and percentages. Univariate analysis will be performed for continuous variables and a $\chi^{2}$ or Fisher's exact test will be applied for categorical variables. Correlation between variables will be assessed with the Pearson or Spearman test. A p value of $<0.05$ will be considered significant.

\section{ETHICS AND DISSEMINATION}

The protocol was approved by the Clinical Research and Laboratory Animal Ethics Committee of the First Affiliated Hospital, Sun Yat-sen University ((2017)286) and all subjects will provide their written informed consent. For all participating hospitals and study sites, approval by the boards of management will also be obtained. Procedures followed were in accord with the ethical standards of the Declaration of Helsinki. The study protocol was registered at www.chictr.org.cn prior to trial commencement.

Changes to the study protocol are documented in amendments. Amendments are submitted for approval to the ethics committee. Major changes will be updated on the trial registration website (www.chictr.org.cn).

After completion, the results will be published in a peer-reviewed journal.

\section{DISCUSSION}

Hypercholesterolaemia is one of the leading modified risk factors of ASCVD and reduction of LDL-C greatly improves patients' clinical outcomes in both primary and secondary prevention. However, the efficacy and adherence of lipid-lowering therapy is far from satisfactory. A transition to a simplified dosing regimen may favourably affect compliance, a major factor that contributes to patient well-being. Once-daily formulations may lead to improved medication adherence, better lipid control as well as potential better outcomes. The APEX study is specifically designed to address whether once per day dosing of XZK is non-inferior to standard two times per day regimen in lipid-lowering efficacy in hyperlipidaemia patients. To our knowledge, this is the first study to evaluate the efficacy of XZK prescribed in different frequencies.

Recent guidelines recommend different LDL-C goals based on the calculated CV risks. ${ }^{4}$ For patients with low to moderate CV risks, lifestyle changes as well as addition of lipid-lowering dietary supplements could be considered. ${ }^{4}$ XZK, produced by the fermentation of RYR, is classified as prescription drug instead of dietary supplement in China. It contains several types of monacolins including monacolin $\mathrm{K}$, which is structurally identical to lovastatin. ${ }^{23}$ A large number of clinical studies have confirmed the lipid-lowering efficacy of XZK and RYR. ${ }^{24-27}$ Therefore, RYR with an active daily dose of 3-10 mg (monacolin K) is a class I recommendation for patients with mild to moderate hypercholesterolaemia and those with statin intolerance. ${ }^{28}{ }^{29}$ However, there are still controversies regarding the safety of RYR. Several cases reports have highlighted the potential adverse reactions of RYR including muscle and hepatic injuries. ${ }^{30}$ Therefore, recently European Food Safety Authority opinion on the safety of monacolins in RYR suggested the dose of monacolins in RYR to be $\leq 3 \mathrm{mg} .{ }^{32}$ However, in fact, it is unclear whether the observed adverse effects in these cases were associated with monacolins or other components or drug interaction. In this case, a meta-analysis including 53 
RCTs and 8535 patients was conducted and confirmed that RYR-based therapy was safe and very well tolerated independent of dose and duration of therapy and population investigated. ${ }^{33}$ Besides, RYR was associated with reduced risk of non-musculoskeletal disorders and severe adverse events. ${ }^{33}$ Therefore, monacolin $\mathrm{K}$ administrated at a dose of $10 \mathrm{mg}$ in our study is overall tolerable and safe. Nevertheless, high quality clinical trials are still needed to further evaluate the safety.

\section{LIMITATIONS}

There are still some limitations in our study. First, the medication duration in our study is only 8 weeks, which may be a little short. However, according to the published clinical trials, XZK showed a significant lipid-lowering effect at a follow-up period of 6-24 weeks. ${ }^{26}$ Besides, our study adopted an open-label design which had its inner bias. However, the open-label design gives us the opportunity to study the possible influence of compliance on the efficacy of the treatment. Third, XZK contains a family of naturally occurring statins besides monacolin $\mathrm{K}$. The lipid-lowering efficacy of every single ingredient within XZK can hardly be evaluated in our study. Further studies are still need to fully characterise the effects of the diverse chemical constituents and the toxicological actions of the bioactive compounds in XZK.

\section{Author affiliations}

${ }^{1}$ Department of Cardiology, The First Affiliated Hospital, Sun Yat-sen University, Guangzhou, China

${ }^{2}$ NHC Key Laboratory of Assisted Circulation, Sun Yat-sen University, Guangzhou, China

${ }^{3}$ Department of Cardiology, The First Affiliated Hospital of Guangzhou Medical University, Guangzhou, China

${ }^{4}$ Department of Cardiology, The Eastern Hospital of the First Affiliated Hospital, Sun Yat-sen University, Guangzhou, China

${ }^{5}$ Department of Cardiology, Central Hospital of Panyu District, Guangzhou, China

Contributors All the authors contributed to the design and development of the study protocol, and have reviewed the manuscript. YD and YC conceived and instructed the study. ZW and JJ designed the study. ZW also provided statistical expertise and supported the development of the statistical analysis plan. DW, AC, $\mathrm{D}-\mathrm{DZ}$ and $\mathrm{JL}$ contributed to recruitment, trial oversight, intervention implementation as well as the follow-up of the subjects.

Funding The APEX study is sponsored by Beijing Beida Weixin Biotechnology Co. Competing interests None declared.

Patient and public involvement Patients and/or the public were not involved in the design, or conduct, or reporting, or dissemination plans of this research.

Patient consent for publication Not required.

Provenance and peer review Not commissioned; externally peer reviewed.

Open access This is an open access article distributed in accordance with the Creative Commons Attribution Non Commercial (CC BY-NC 4.0) license, which permits others to distribute, remix, adapt, build upon this work noncommercially, and license their derivative works on different terms, provided the original work is properly cited, appropriate credit is given, any changes made indicated, and the use is non-commercial. See: http://creativecommons.org/ licenses/by-nc/4.0/.

\section{ORCID iD}

Yili Chen http://orcid.org/0000-0001-9999-9413
REFERENCES

1 WM Centre. Cardiovascular diseases, 2017. Available: http:// wwwwhoint/cardiovascular_diseases/en/

2 Stone NJ, Robinson JG, Lichtenstein AH, et al. 2013 ACC/ AHA guideline on the treatment of blood cholesterol to reduce atherosclerotic cardiovascular risk in adults: a report of the American College of Cardiology/American heart association Task force on practice guidelines. J Am Coll Cardiol 2014;63:2889-934.

3 Cholesterol Treatment Trialists' (CTT) Collaboration, Baigent C, Blackwell L, et al. Efficacy and safety of more intensive lowering of LDL cholesterol: a meta-analysis of data from 170,000 participants in 26 randomised trials. Lancet 2010;376:1670-81.

4 Mach F, Baigent C, Catapano AL, et al. 2019 ESC/EAS guidelines for the management of dyslipidaemias: lipid modification to reduce cardiovascular risk. Eur Heart J 2020;41:111-88.

5 Sirtori CR, Mombelli G, Triolo M, et al. Clinical response to statins: mechanism(s) of variable activity and adverse effects. Ann Med 2012;44:419-32.

6 Danese MD, Gleeson M, Kutikova L, et al. Management of lipidlowering therapy in patients with cardiovascular events in the UK: a retrospective cohort study. BMJ Open 2017;7:e013851.

7 De Vera MA, Bhole V, Burns LC, et al. Impact of statin adherence on cardiovascular disease and mortality outcomes: a systematic review. Br J Clin Pharmacol 2014;78:684-98.

8 Heber D, Yip I, Ashley JM, et al. Cholesterol-Lowering effects of a proprietary Chinese red-yeast-rice dietary supplement. Am J Clin Nutr 1999;69:231-6.

9 Zhao SP, Liu L, Cheng YC, et al. Xuezhikang, an extract of Cholestin, protects endothelial function through antiinflammatory and lipidlowering mechanisms in patients with coronary heart disease. Circulation 2004;110:915-20.

10 Li Z, Seeram NP, Lee R, et al. Plasma clearance of lovastatin versus Chinese red yeast rice in healthy volunteers. J Altern Complement Med 2005;11:1031-8.

11 Moriarty PM, Roth EM, Karns A, et al. Effects of Xuezhikang in patients with dyslipidemia: a multicenter, randomized, placebocontrolled study. J Clin Lipidol 2014;8:568-75.

12 Srivastava K, Arora A, Kataria A, et al. Impact of reducing dosing frequency on adherence to oral therapies: a literature review and meta-analysis. Patient Prefer Adherence 2013;7:419-34.

13 Yumuk V, Tsigos C, Fried M, et al. European guidelines for obesity management in adults. Obes Facts 2015;8:402-24.

14 Sharifi $\mathrm{MH}$, Eftekhari $\mathrm{MH}$, Ostovan MA, et al. Effects of a therapeutic lifestyle change diet and supplementation with Q10 plus L-carnitine on quality of life in patients with myocardial infarction: a randomized clinical trial. J Cardiovasc Thorac Res 2017;9:21-8.

15 Lin C-C, Li T-C, Lai M-M. Efficacy and safety of Monascus purpureus went rice in subjects with hyperlipidemia. Eur $J$ Endocrinol 2005;153:679-86.

03f1785a-2d28-4dae-b27a-3d732f426560 Pundir CS, Minakshi. Coimmobilization of lipase, glycerol kinase, glycerol-3-phosphate oxidase and peroxidase on to aryl amine glass beads affixed on plastic strip for determination of triglycerides in serum. Indian $J$ Biochem Biophys 2008;45:111-6.

17 Newman CB, Preiss D, Tobert JA, et al. Statin safety and associated adverse events: a scientific statement from the American heart association. Arterioscler Thromb Vasc Biol 2019;39:e38-81.

18 Penson PE, Mancini GBJ, Toth PP, et al. Introducing the 'Drucebo' effect in statin therapy: a systematic review of studies comparing reported rates of statin-associated muscle symptoms, under blinded and open-label conditions. J Cachexia Sarcopenia Muscle 2018;9:1023-33.

19 Rosenson RS, Baker S, Banach M, et al. Optimizing cholesterol treatment in patients with muscle complaints. J Am Coll Cardiol 2017;70:1290-301.

20 Baber N. International Conference on harmonisation of technical requirements for registration of pharmaceuticals for human use $(\mathrm{ICH})$. Br J Clin Pharmacol 1994;37:401-4

21 Zhian J, Jing X, Weijiang Z, et al. Clinical observation of Xuezhikang in the treatment of hyperlipidemia. Cap Med 1998;03:38-9.

22 Ambery P, Stylianou A, Atkinson G, et al. Open-Label randomized non-inferiority trial of a fixed-dose combination of glimepiride and atorvastatin for the treatment of people whose type 2 diabetes is uncontrolled on metformin. Diabet Med 2016;33:1084-93.

23 Zhu B, Qi F, Wu J, et al. Red yeast rice: a systematic review of the traditional uses, chemistry, pharmacology, and quality control of an important Chinese folk medicine. Front Pharmacol 2019;10:1449.

24 Liu J, Zhang J, Shi Y, et al. Chinese red yeast rice (Monascus purpureus) for primary hyperlipidemia: a meta-analysis of randomized controlled trials. Chin Med 2006;1:4. 
25 Lu Z, Kou W, Du B, et al. Effect of Xuezhikang, an extract from red yeast Chinese rice, on coronary events in a Chinese population with previous myocardial infarction. Am J Cardiol 2008;101:1689-93.

26 Gerards MC, Terlou RJ, Yu H, et al. Traditional Chinese lipid-lowering agent red yeast rice results in significant LDL reduction but safety is uncertain - a systematic review and meta-analysis. Atherosclerosis 2015;240:415-23.

27 Banach M, Bruckert E, Descamps OS, et al. The role of red yeast rice (RyR) supplementation in plasma cholesterol control: a review and expert opinion. Atheroscler Supp/ 2019;39:e1-8.

28 Cicero AFG, Colletti A, Bajraktari G, et al. Lipid lowering nutraceuticals in clinical practice: position paper from an international lipid expert panel. Arch Med Sci 2017;13:965-1005
29 Banach M, Patti AM, Giglio RV, et al. The Role of Nutraceuticals in Statin Intolerant Patients. J Am Coll Cardiol 2018;72:96-118.

30 Mazzanti G, Moro PA, Raschi E, et al. Adverse reactions to dietary supplements containing red yeast rice: assessment of cases from the Italian surveillance system. Br J Clin Pharmacol 2017;83:894-908.

31 Raschi E, Girardi A, Poluzzi E, et al. Adverse events to food supplements containing red yeast rice: comparative analysis of FAERS and CAERS reporting systems. Drug Saf 2018;41:745-52.

32 EFSA ANS Panel (EFSA Panel Food Additives and Nutrient Sources added to Food. Scientific opinion on the safety of monacolins in red yeast rice. EFSA J 2018;16:5368.

33 Fogacci F, Banach M, Mikhailidis DP, et al. Safety of red yeast rice supplementation: a systematic review and meta-analysis of randomized controlled trials. Pharmacol Res 2019;143:1-16. 